\author{
Z. Holub - A. Jabor · J. Lukac $\cdot$ L. Kliment \\ S. Urbanek · E. Shibalova
}

\title{
Laparoscopic-assisted vaginal myomectomy: a feasibility study
}

Published online: 18 June 2005

(C) Springer-Verlag Berlin / Heidelberg 2005

\begin{abstract}
This pilot study assessed the feasibility and complications of a combined laparoscopic and vaginal approach through the posterior colpotomy in cases of fundal or posterior wall uterine fibroids. Twenty-four women with single symptomatic fibroids (size 5-8 cm) were prospectively assigned to undergo either laparoscopic-assisted vaginal myomectomy (LAVM; $n=12)$ or laparoscopic myomectomy (LM; $n=12)$. Surgical characteristics, hospital stay, and complications were analyzed. Blood samples for assay of tissue markers [C-reactive protein (CRP), creatine kinase (CK), and white blood cell count] and hemoglobin were taken preoperatively and on the 1 st and $3 \mathrm{rd}$ postoperative days. There were no differences between the two groups with regard to demographic characteristics. The mean operating time was shorter in the LAVM group $(64.44 \pm 17.4 \mathrm{~min}$ vs. $89.00 \pm 23.4 \mathrm{~min}, P<0.05)$. There was no difference in blood loss or complications between the groups. One woman in the LM group required vaginal conversion for an unsuccessful hemostasis during laparoscopic coagulation. The difference in decline of hemoglobin and tissue marker levels was statistically insignificant. Only the serum CRP level was statistically different between the groups. This may indicate that the local abdominal wall injury in the LM group was influenced by minilaparotomy in two cases of larger fibroids. In both groups, serum CRP was significantly
\end{abstract}

\footnotetext{
Z. Holub $(\bowtie) \cdot$ J. Lukac · L. Kliment · S. Urbanek E. Shibalova

Department of Obstetrics and Gynecology,

Endoscopic Training Centre,

Baby Friendly Hospital,

Vancurova 1548, 27258

Kladno, Czech Republic

E-mail: holubz@seznam.cz

Tel.: + 420-312-606383

Fax: +420-312-606417
}

A. Jabor

Department of Biochemistry, Baby Friendly Hospital, Vancurova 1548, 27258 Kladno, Czech Republic increased when blood loss was greater than $200 \mathrm{ml}$. In conclusion, LAVM is a feasible minimally invasive procedure and has also been associated with a shorter operating time compared with LM. A combined laparoscopic and vaginal approach in dealing with fundal and/or posterior wall uterine fibroids through the posterior cul-de-sac is an alternative to either pure laparoscopic myomectomy or laparoscopic-assisted myomectomy.

Keywords Uterine fibroids - Myomectomy · Laparoscopy $\cdot$ Posterior colpotomy

\section{Introduction}

Laparoscopic-assisted vaginal myomectomy (LAVM) is a minimally invasive surgical procedure for removing uterine myomas. It was first described in the late 1990s by Goldfarb and Fanarjian [1] and Pelosi and Pelosi [2]. These authors independently worked on a variant of laparoscopic myomectomy (LM) in which the dominant myoma was removed laparoscopically and the uterus was delivered via colpotomy into the vagina for removal of secondary uterine myomas and for uterine closure [1].

The aim of this pilot study was to compare the feasibility and complications of LAVM and LM. The study is part of a prospective Czech multicenter study, Fibroid Surgery Alternatives Treatment (FSAT), which is supported by the Grant Foundation of the Minister of Health of the Czech Republic (NR-7982-3/2004).

\section{Materials and methods}

From January 2003 to June 2004, we conducted a pilot study involving 24 patients with single symptomatic uterine myomas. Each woman gave her informed consent to be included in the study, which had been previously approved by the Hospital Kladno's local ethics 
committee. Transvaginal ultrasound and/or magnetic resonance imaging were used to obtain information on the number, size, and location of the myomas. Inclusion criteria for entering the study were age 25-45 years, normal hemoglobin level, and the presence of a single symptomatic intramural or subserous posterior or fundal-posterior fibroid with a total diameter of 5-8 cm. Women who wished to achieve pregnancy or patients with submucous fibroids were excluded. The first 12 women who met the inclusion criteria underwent LM or laparoscopic-assisted myomectomy (LAM), and the subsequent 12 women underwent LAVM.

All patients had normal preoperative follicle stimulating hormone, luteinizing hormone, $17 \beta$-estradiol, Creactive protein (CRP), and creatine kinase (CK) levels and normal white blood cell (WBC) counts, and they had no significant concomitant disease. Duration of the surgical procedure was calculated from the first skin incision to the last skin suture. Intraoperative blood loss was measured as blood accumulated in the aspirator during the operation. Postoperative blood loss was approximated from the postoperative fluid collection using a Redon catheter. Postoperative fever was defined as a body temperature of a least $38^{\circ} \mathrm{C}$ on two consecutive measurements at least $6 \mathrm{~h}$ apart, excluding the first $24 \mathrm{~h}$. The incidence of intraoperative and postoperative complications and fever, analgesic requirements, and the length of the postoperative hospital stay were recorded. Patients were seen 3 months after surgery. Patient demographic characteristics and short-term postoperative outcomes were obtained from hospital and outpatient medical records.

Prophylaxis consisted of a single dose of ampicillin $(2.0 \mathrm{~g})$ and anticoagulant therapy with low-molecularweight heparin. Gonadotropin-releasing hormone (GnRH) agonist pretreatment was not used in selected cases. Blood samples for hemoglobin and assay of markers of inflammatory response and tissue trauma (CRP, CK, and WBCs) were taken preoperatively and on the 1 st and 3 rd postoperative days.

\section{Statistical analysis}

Statistical analysis was performed with the MannWhitney $U$-test for unpaired results. Differences within groups were analyzed by means of the paired Wilcoxon test for two related samples. Data are presented as mean \pm SD and interquartile range $(25-75 \%)$ or as a number or percentage. Statistical significance was defined as $P<0.05$.

\section{Operative procedure}

Laparoscopy was done under general anesthesia in the lithotomy position using video monitoring equipment. The telescope was inserted subumbilically, and one $11-\mathrm{mm}$ port was made suprapubically and medially. Two 5-mm ports were placed in each of the lower quadrants at the lateral edge of the rectus abdominis muscle.

\section{Laparoscopic-assisted vaginal myomectomy}

A routine laparoscopic myomectomy using a harmonic scalpel was performed. A 5-mm myoma screw was inserted into the myoma, and a grasper with locking mechanism was placed on the inferior edge of the wound. The myoma screw was used to direct the myoma toward the cul-de-sac. Posterior colpotomy was then performed in the routine manner. A 5-mm grasper was used to guide the uterus to the colpotomy site. The myoma was grasped with a tenaculum and removed vaginally. The clamps were placed on the edges of the wounds, and the fundus of the uterus was delivered, via the posterior colpotomy incision, into the vagina. The uterine incision was sutured in one to two layers. The repaired uterus was returned to the abdominal cavity, and the colpotomy incision was closed. After a laparoscopic final check to control hemostasis, a Redon catheter was left for drainage.

\section{Laparoscopic myomectomy}

An incision was made through the uterine wall and the pseudocapsule of the fibroid. Traction was applied to the fibroid, along with harmonic scalpel dissection, in order to cleave the fibroid. After complete fibroid removal using the Steiner electric morcellator (Storz, Tuttlingen, Germany), the edges of the uterine defect were approximated using ultrasonically activated shears (LCS-K5 or LCS-C, UltraCision; Ethicon EndoSurgery, Johnson \& Johnson, Cincinnati), coagulating the superficial myometrium without suturing. If the defect was deep or large (over $2 \mathrm{~cm}$ ), the uterine incision, including myometrium and serosa, was closed with one or two layers of interrupted absorbable sutures of $1-0$ or $2-0$ polyglactin (Vicryl; Ethicon, Edinburgh). In cases of posterior fibroid location in which it was impossible to achieve hemostasis or perfect sutures, the LAM operative technique was used. This modification of LM was introduced and described in more detail by Nezhat et al. [3]. After a final check to control hemostasis, a Redon catheter was left for drainage. Prophylaxis consisted of a single dose of ampicillin (2.0 g) and anticoagulant therapy with low-molecular-weight heparin.

\section{Results}

The patients' demographic characteristics and indications for surgery are shown in Table 1. All patients underwent a successful operative procedure and completed follow-up at 3 months. There were no significant differences between the two groups in terms of age, weight, previous surgery, size of fibroids, indication for 
Table 1 Patient and fibroid characteristics in the laparoscopic-assisted vaginal myomectomy (LAVM) and laparoscopic myomectomy (LM) groups ( $N S$ not significant)

\begin{tabular}{llll}
\hline & LAVM group $(n=12)$ & LM group $(n=12)$ & $P<$ value \\
\hline Mean age in years (range) & $35.00(30.00-38.75)$ & $36.50(29.00-41.00)$ & NS \\
Mean weight in kg (range) & $77.20(53.00-81.90)$ & $77.60(56.30-84.00)$ & NS \\
Previous pelvic surgery $(n)$ & $3(30 \%)$ & $2(20 \%)$ & NS \\
Fibroid & & & \\
Mean maximum diameter in mm & $57.66 \pm 6.24$ & $64.50 \pm 11.65$ & $\mathrm{NS}$ \\
Fundal-posterior location & 4 & 4 & $\mathrm{NS}$ \\
Posterior location & 8 & 8 & $\mathrm{NS}$ \\
Indications $(n)$ & & & $\mathrm{NS}$ \\
Menorrhagia & 7 & 6 & $\mathrm{NS}$ \\
Pelvic pain & 4 & 3 & $\mathrm{NS}$ \\
Urinary frequency & 1 & 3 & \\
\hline
\end{tabular}

surgery, tissue markers, or hemoglobin level before myomectomy. The most common indication for myomectomy was menorrhagia, but more than half of the patients had more than one indication. The mean operating time was shorter in the LAVM group $(64.44 \pm 17.4 \mathrm{~min}$ vs. $89.00 \pm 23.4 \mathrm{~min} ; \quad P<0.05$; Table 2). There was no difference between the groups in mean \pm SD intraoperative or postoperative blood loss or in the hemoglobin fall on days 1 and 3 after surgery. Typical LM was performed in nine cases and LAM in two women who had $8-\mathrm{cm}$ fibroids. Conversion to LAVM was required in one woman in the LM group with an $8-\mathrm{cm}$ distal posterior fibroid in whom it was impossible to achieve hemostasis by coagulation. Blood transfusion was required in this case. This patient was included in the statistical analysis of LM and calculated only as a complication. No damage to the uterine cavity or fallopian tube was observed in any patient. There were no other major intraoperative or postoperative complications. No differences in the length of hospital stay or the time required to return to normal activity were found.

Concerning the tissue marker results, preoperative levels of the studied markers were low in the comparison groups, and the circulating concentrations of CRP and WBCs increased significantly after surgery in both groups (Table 3). Only the serum CRP level was statistically different between the groups. In both groups, serum CRP was significantly increased in the patient in whom blood loss was more than $200 \mathrm{ml}$.

\section{Discussion}

The results of this study confirm the feasibility of LAVM, which has been previously described in other

Table 2 Surgical results in the laparoscopic-assisted vaginal myomectomy (LAVM) and laparoscopic myomectomy (LM) groups (NS not significant, EPBL estimated postoperative blood loss)

\begin{tabular}{llll}
\hline Parameter & LAVM group $(n=12)$ & LM group $(n=11)^{\mathrm{a}}$ & $P$-value \\
\hline Intraoperative blood loss $(\mathrm{ml})$ & $121.11 \pm 102.03(45-157.5)$ & $195.00 \pm 114.13(100-250)$ & NS \\
EPBL (ml) & $47.22 \pm 62.20(10-57.50)$ & $59.50 \pm 73.99(5-50)$ & NS \\
Operating time (min) & $64.44 \pm 17.40$ & $89.00 \pm 23.42$ & $P<0.05$ \\
Hospital stay (days) & $2.6 \pm 0.3$ & $2.4 \pm 0.2$ & NS \\
Total complications $(n)$ & 1 & 1 & NS \\
Transfusion & 0 & 0 & \\
Preperitoneal emphysema & 1 & & \\
\hline
\end{tabular}

${ }^{\mathrm{a}}$ The conversion case was not calculated

Table 3 Results of hemoglobin and tissue markers in the laparoscopic-assisted vaginal myomectomy (LAVM) and laparoscopic myomectomy $(\mathrm{LM})$ groups ( $P O D$ postoperative day, $N S$ not significant)

\begin{tabular}{lllll}
\hline Parameter & POD & LAVM group $(n=12)$ & LM group $(n=11)$ & $P$-value \\
\hline Basal hemoglobin $(\mathrm{g} / \mathrm{dl})$ & 0 & $13.13 \pm 1.24$ & $13.59 \pm 0.82$ & $\mathrm{NS}$ \\
& 1 & $11.77 \pm 1.62$ & $12.23 \pm 0.84$ & $\mathrm{NS}$ \\
& 3 & $11.31 \pm 1.60$ & $12.03 \pm 1.09$ & $\mathrm{NS}$ \\
C-reactive protein $(\mathrm{mg} / \mathrm{l})$ & 0 & $<5.00$ & 5.53 & $\mathrm{NS}$ \\
& 1 & 12.75 & 22.25 & $P<0.05$ \\
& 3 & 21.60 & 33.00 & $P<0.05$ \\
Creatine kinase $(\mu \mathrm{kat} / \mathrm{l})$ & 0 & 1.31 & 1.10 & $\mathrm{NS}$ \\
& 1 & 3.99 & 3.52 & $\mathrm{NS}$ \\
& 3 & 2.90 & 1.45 & $\mathrm{NS}$ \\
White blood cells $\left(10^{9} / 1\right)$ & 0 & 7.48 & 5.88 & $\mathrm{NS}$ \\
& 1 & 9.62 & 8.85 & $\mathrm{NS}$ \\
& 3 & 7.60 & 6.00 & $\mathrm{NS}$ \\
\hline
\end{tabular}


reports $[1,2,4]$. Despite the small number of patients in our pilot study, the operating time was significantly shorter with LAVM than with LM. These results are in harmony with previous studies comparing vaginal and laparoscopic myomectomy or hysterectomy [5-7]. No case of damage to the fallopian tube or opening of the uterine cavity was observed. Like other laparoscopic procedures, LAVM offers a shorter hospital stay relative to the abdominal approach. No differences in tissue markers or complication rates were found between the studied groups.

In our study, there was no difference between the groups with regard to hospital stay, which was similar to the results reported in previous LAVM series $[1,4]$. We did not observe the occurrence of postsurgical urine retention or transient macroscopic hematuria; this fact was different from other studies where posterior or anterior colpotomy was used [1,8].

Furthermore, this study shows that LM is also feasible in selected patients with single fundal or posterior intramural fibroids. However, one of the 12 patients in the LM group with a large posterior fibroid required conversion to LAVM for intraoperative bleeding, which was successfully treated by the vaginal route.

In 1994, Nezhat et al. [3] described LAM, a procedure that combines operative laparoscopy and minilaparotomy $(4-5 \mathrm{~cm})$ for removing single and multiple fibroids. In their retrospective study of 57 patients, with fibroids ranging from 28 to $988 \mathrm{~g}$, the authors reported a mean operating time of $127 \mathrm{~min}$. Three major objectives of LAM were mentioned: minimizing blood loss, preventing postoperative adhesions, and maintaining uterine wall integrity. In addition, comparing the procedure with LM, they concluded that LAM is technically less difficult, allows better closure of the uterine defect, and may require less time to carry out. The importance of fibroid location was not studied and compared between LM and LAM.

Koh and Janik [9] have lamented the impossibility of achieving full visual control in the course of myoma enucleation in LAM or LAVM. These authors suggested that in both LAM and LAVM, exteriorizing and handling the uterus reproduces the very factors that cause de novo adhesion formation or introduce infection. Agostini et al. [5] reported Gardnerella vaginalis bacteremia after vaginal myomectomy assisted by diagnostic laparoscopy. We suggest that the risk of postoperative infection in combined laparovaginal procedures can be decreased by intraoperative antibiotic administration and bacteriological vaginal examination. Women at risk are those with a history of sexually transmitted infection or chronic vaginitis, and they should be treated preventively with metronidazol or excluded from LAVM.

Wang et al. [4] have described LAVM. A suture guide showing the site of the fibroid was done by laparoscopy and exteriorized by colpotomy. This guide allows easier repair and traction of fibroids. From our experience, it is advantageous to perform only incomplete fibroid laparoscopic excision and to retain the bridge guide or stalk in the distal margin. From our point of view and in accordance with Goldfarb and Fanarjian [1], the LAVM procedure offers advantages over both LM and LAM. Compared with LM, LAVM provides the control and safety of direct suturing in addition to having the advantages of digital palpation to detect less obvious fibroids. Compared with LAM, LAVM requires a smaller abdominal incision and avoids cutting through several layers of the abdominal wall.

In conclusion, we confirm that LAVM is feasible and safe. LM is a technically demanding procedure requiring special skills, such as the ability to perform laparoscopic suturing $[10,11]$. LAVM or LAM may offer better management by decreasing these technical demands, as they allow exact suturing of the uterine defect in layers. Moreover, LAVM has been associated with a significantly shorter operating time than LM. We suggest that LAVM can substitute for LM in cases of posterior fibroids. Randomized studies are necessary to compare the approaches for myomectomy: the combined laparovaginal approach, the vaginal route, laparoscopy, and laparotomy.

Acknowledgments This work was supported by the Grant Foundation of the Minister of Health of the Czech Republic (NR 7982$3 / 2004)$.

\section{References}

1. Goldfarb HA, Fanarjian NJ (2001) Laparoscopic-assisted vaginal myomectomy: a case report and literature review. JSLS 5:81-86

2. Pelosi M III, Pelosi M (1997) Laparoscopic-assisted transvaginal myomectomy. J Am Assoc Gynecol Laparosc 4:241-246

3. Nezhat C, Nezhat F, Bess O, Nezhat C, Mashiach R (1994) Laparoscopically assisted myomectomy: a report of a new technique in 57 cases. Int $\mathbf{J}$ Fertil 39:39-44

4. Wang CJ, Yen CF, Lee CL, Soong YK (2000) Laparoscopicassisted vaginal myomectomy. J Am Assoc Gynecol Laparosc 7:510-514

5. Agostini A, Deval B, Birsan A et al (2004) Vaginal myomectomy using posterior colpotomy: feasibility in normal practice. Eur J Obstet Gynecol Reprod Biol 116:217-220

6. Birsan A, Deval B, Poncelet Ch, Darai E (2003) Vaginal and laparoscopic myomectomy for large posterior myomas: results of a pilot study. Eur J Obstet Gynecol Reprod Biol 110:89-93

7. Darai E, Soriano D, Kimata C, Laplace C, Lecuru F (2001) Vaginal hysterectomy for enlarged uteri, with or without laparoscopic assistance: randomized study. Obstet Gynecol 97:712-716

8. Chin HY, Lee CL, Yen CF et al (2004) Laparoscopic-assisted vaginal myomectomy through an anterior approach. J Laparoendosc Adv Surg Tech 14:135-138

9. Koh Ch, Janik G (2003) Laparoscopic myomectomy: the current status. Curr Opin Obstet Gynecol 15:295-301

10. Signorile PG (2002) Laparoscopic-ultraminilaparotomic myomectomy LUM-laparoscopic-ultraminilaparotomic embolized myomectomy (LUEM). Surgical techniques. Clin Exp Obstet Gynecol 29:277-280

11. Cagnaci A, Pirrilo D, Malmusi S, Arangnio S, Alessandrini C, Volpe A (2003) Early outcome of myomectomy by laparotomy, minilaparotomy and laparoscopically-assisted minilaparotomy. A randomized prospective study. Hum Reprod 12:2590-2594 